\title{
Rotation velocities of hot horizontal branch stars in the globular clusters NGC 1904, NGC 2808, NGC 6093, and NGC 7078: The database $e^{\star, \star \star}$
}

\author{
A. Recio-Blanco ${ }^{1}$, G. Piotto ${ }^{1}$, A. Aparicio ${ }^{2}$, and A. Renzini ${ }^{3}$ \\ 1 Dipartimento di Astronomia, Università di Padova, Vicolo dell'Osservatorio 2, 35122 Padova, Italy \\ e-mail: recio@pd.astro.it, piotto@pd.astro.it \\ 2 Instituto de Astrofisica de Canarias, via Lactea s/n, 382002 La Laguna Tenerife, Spain \\ e-mail: aaj@ll.iac.es \\ 3 ESO, Karl-Schwarzschild-Str. 2, 85748 Garching bei München, Germany \\ e-mail: arenzini@eso.org
}

Received 9 May 2003 / Accepted 19 December 2003

\begin{abstract}
We present radial and rotation $(v \sin i)$ velocity measurements from UVES+VLT high resolution spectra of 61 stars in the blue horizontal branches (HB) of the Galactic globular clusters NGC 1904 (M 79), NGC 2808, NGC 6093 (M 80) and NGC 7078 (M 15).

The data reduction and the velocity determination, based on cross-correlation techniques, are discussed in detail. Most of this database has been used by Recio-Blanco et al. (2002) in their analysis of the rotation velocity properties of blue HB stars. Here we present additional data for NGC 2808. We confirm the results of the previous paper, in particular, a possible link between the HB jump and the abrupt change in the rotational velocity distribution around $T_{\text {eff }} \sim 11500 \mathrm{~K}$.
\end{abstract}

Key words. galaxy: globular clusters: general - stars: horizontal-branch - stars: rotation

\section{Introduction}

The high resolution spectroscopic capabilities of the Ultraviolet Visual Echelle Spectrograph (UVES), combined with the collecting power of the Very Large Telescope (VLT), offer an exceptional opportunity to study the nature of the hot stars populating the anomalously extended blue horizontal branches (HB) in Galactic globular clusters (GC). The horizontal branches with blue tails (BT, here considered to host stars hotter than $12000 \mathrm{~K}$ ) probably represent the most extreme of the mixed bag of $\mathrm{HB}$ anomalies lumped under the term "secondparameter problem" (cf. Catelan et al. 1998; Piotto et al. 1999 for a more detailed discussion). We already know that BTs host $\mathrm{He}$-core $\mathrm{H}$-shell burning stars which have lost up to a factor of two more mass during the Red Giant Branch (RGB) ascent, than other HB stars in the same cluster (D'Cruz et al. 1996). However, we still lack of an explanation for such a sizable mass loss. Several mechanisms, regulating either the core mass or the

Send offprint requests to: A. Recio-Blanco,

e-mail: recio@pd.astro.it

* Based on observations with the ESO Very Large Telescope + UVES, at the Paranal Observatory, Chile.

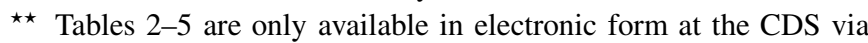
anonymous ftp to cdsarc.u-strasbg.fr (130.79.128.5) or via http://cdsweb.u-strasbg.fr/cgi-bin/qcat?]/A+A/417/597 amount of mass loss that a star experiences on the upper RGB have been proposed as possible second parameter candidates. Among them, stellar rotation, which would delay the helium core flash in a red giant, was also suggested (Mengel \& Gross 1976; Renzini 1977; Peterson et al. 1995). A faster rotating star would therefore increase its helium core mass and would experience a higher mass loss rate in the RGB, leading to bluer and brighter HB star.

The first investigations on the rotation velocity of blue horizontal branch stars were done by Peterson and collaborators (Peterson 1983, 1985a, 1985b; Peterson et al. 1995). Some of the blue HB stars in the BT cluster M 13 were found to be rotating as fast as $40 \mathrm{~km} \mathrm{~s}^{-1}$. On the contrary, clusters with cooler HB morphologies, as M 3 and NGC 288, showed only values of $v \sin i$ slower than about $20 \mathrm{~km} \mathrm{~s}^{-1}$. More recently, Behr et al. (2000a, B00a) have suggested the existence of a discontinuity in stellar rotation velocity across one region underpopulated of stars (gap), at $T_{\text {eff }} \simeq 11000 \mathrm{~K}$, in the HB of M 13 . Bluewards of the gap, all the stars showed modest rotations ( $v \sin i<10 \mathrm{~km} \mathrm{~s}^{-1}$ ), while, to the red side of the gap, several rapidly rotating stars were found. A similar discontinuity was also detected in M 15 (Behr et al. 2000b, B00b).

UVES+VLT allows us to measure the rotational velocity of blue HB stars as faint as $V=18$ with an accuracy of a few $\mathrm{km} \mathrm{s}^{-1}$. For this reason, and prompted by the previous 

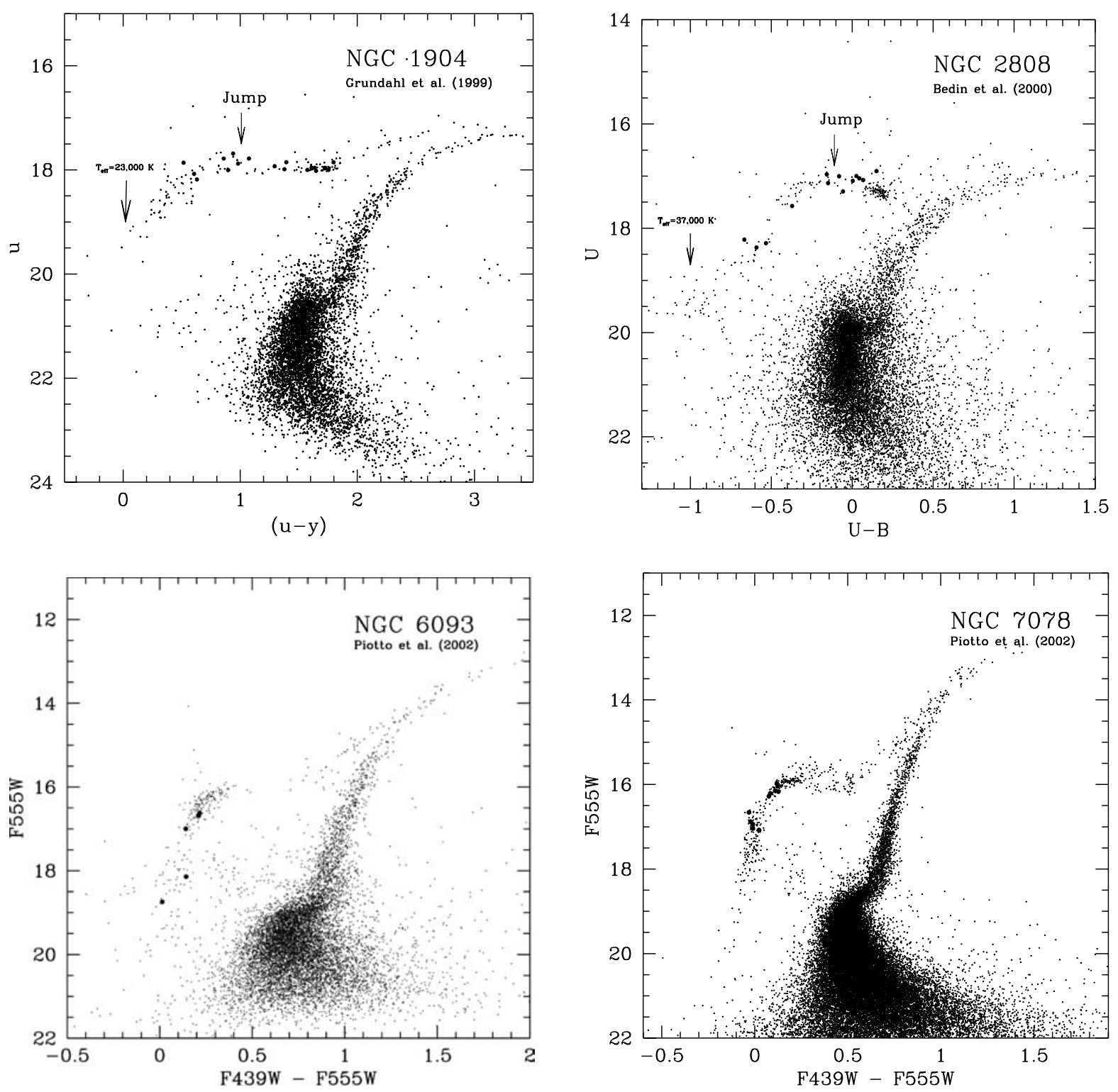

Fig. 1. The target cluster CMDs with their corresponding selected stars.

Table 1. The target clusters parameters. $[\mathrm{Fe} / \mathrm{H}]$ is from Carretta et al. (2001). The absolute visual magnitude, distance modulus, central concentration and Galactocentric distance are taken from Harris (1999).

\begin{tabular}{ccccccc}
\hline \hline Cluster & {$[\mathrm{Fe} / \mathrm{H}]$} & $M_{V}$ & $(m-M)_{V}$ & $E(B-V)$ & $\log \left(r_{t} / r_{c}\right)$ & $R_{\mathrm{GC}}(\mathrm{Kpc})$ \\
\hline NGC 1904 & -1.37 & -7.86 & 15.59 & 0.01 & 1.72 & 18.8 \\
NGC 2808 & -1.21 & -9.36 & 15.56 & 0.23 & 1.77 & 11.0 \\
NGC 6093 & -1.43 & -8.23 & 15.56 & 0.18 & 1.95 & 3.8 \\
NGC 7078 & -2.12 & -9.17 & 15.37 & 0.10 & $2.50 c$ & 10.4 \\
\hline
\end{tabular}

results of B00a, we started a program to investigate how stellar rotation changes as a function of the position along the $\mathrm{HB}$, and from cluster to cluster. We observed at least ten stars per cluster, to statistically avoid the problem of the projection angle $(\sin i)$ in the determination of stellar rotation. At the present time, we have analyzed 61 stars in 4 Galactic GCs: NGC 1904 (M 79), NGC 2808, NGC 6093 (M 80) and NGC 7078 (M 15). All the target clusters have a blue tail HB morphology, although their horizontal branches have different temperature extensions (see their colour-magnitude diagrams in Fig. 1). The HB of M 79 reaches $T_{\text {eff }} \sim 23000 \mathrm{~K}$, both M 80 and M 15 BTs reach a $T_{\text {eff }}$ around $30000 \mathrm{~K}$, while the HB in NGC 2808 goes up to $T_{\text {eff }} \sim 37000 \mathrm{~K}$. They also span a range of metallicity, total luminosity, concentration,... as summarized in Table 1.

The target stars have a temperature in the range $8000 \mathrm{~K}<$ $T_{\text {eff }}<28000 \mathrm{~K}$, distributed on the two sides of the Strömgren $\left(u, u y\right.$ ) luminosity "jump" at $T_{\text {eff }} \simeq 11500 \mathrm{~K}$ (Grundahl et al. 1999, G99). Most of the database we are going to present in 
this paper has already been used by Recio-Blanco et al. (2002, R02), with a number of interesting results. For the first time, we revealed the presence in NGC 1904 of a considerably large number of fast $\left(v \sin i>20 \mathrm{~km} \mathrm{~s}^{-1}\right) \mathrm{HB}$ rotators, and confirmed the fast rotators already detected in M 15 by B00b. In both cases, fast rotators were confined to the cool end of the blue $\mathrm{HB}$, as in $\mathrm{M} \mathrm{13}$. In this paper, we add 5 more stars in NGC 2808, improving statistics on the cool side of the G99 jump for this cluster. We also discuss in detail the reduction and analysis techniques (Sects. 2, 3 and 4). In addition, we present the position and the radial and projected rotational velocities for all the stars so far observed (Sect. 4). A brief discussion on the results (Sect. 5) is also included.

\section{Observations and data reduction}

We selected our targets from three different photometric data sets: the F439W and F555W HST-WFPC2 photometry by Piotto et al. (2002), the ground-based Johnson $V, I$ photometry by Rosenberg et al. (2000), and the Johnson $V, B, U$ data from Bedin et al. (2000). In addition, we have also identified the NGC 1904 targets in the Strömgren $u, y$ photometry by G99.

The observations were carried out with the UVES echelle spectrograph, mounted on the Unit 2 (Kueyen) of the VLT, and the $2 \mathrm{~K} \times 4 \mathrm{~K}, 15 \mu \mathrm{m}$ pixel size blue $\mathrm{CCD}$, with a readout noise of $3.90 \mathrm{e}^{-}$and a conversion factor of $2.04 \mathrm{e}^{-} /$ADU. The UVES blue arm, with a spectral coverage in the 3730$5000 \AA$ range, was used. Combined with a slit width of 1.0 arcsec, we achieved a resolution of $R \sim 40000(\delta \lambda \sim 0.1 \AA$, $\delta v \sim 7.5 \mathrm{~km} \mathrm{~s}^{-1}$, see the UVES user manual by Kaufer et al. 2003). We decided to use only the UVES blue arm because there were very few lines in the red arm spectral range useful for the measurement of stellar rotation, specially for the hottest stars. The spectra were obtained during 3 observing runs: July 30-August 2, 2000, January 19-23, 2001, and March 1-7, 2002 (service mode). The exposure times range from $800 \mathrm{~s}$ (for $V \sim 16$ ) to $8500 \mathrm{~s}$ (for $V \sim 18.5$ ). We generally limited individual exposure times to 1500 or $2000 \mathrm{~s}$, to minimize cosmic ray accumulation, and then coadded 2 to 4 frames per star. The typical signal-to-noise ratios, measured with the task splot within IRAF, are about 10 to 15 per pixel, but in some cases they achieve a value of $S / N \sim 20-30$ (see Tables 2 and 3 for a detailed information). In general, all the targets lie in the low-crowding outskirts of the parent cluster, to avoid contamination from other stars. The isolation of the targets was checked directly, using the corresponding avaliable images of each cluster. During each observing run, we also collected high $S / N$ spectra of a set of field rotational velocity standards (Peterson 1983), with spectral types close to those of our program stars: HD $74721\left(v \sin i<6 \mathrm{~km} \mathrm{~s}^{-1}\right)$, HD $130095(v \sin i<$ $\left.6 \mathrm{~km} \mathrm{~s}^{-1}\right)$, HD $213468\left(v \sin i<10 \mathrm{~km} \mathrm{~s}^{-1}\right)$, HD 117880 $\left(v \sin i=12 \pm 3 \mathrm{~km} \mathrm{~s}^{-1}\right)$, HD $19445\left(v \sin i=13 \pm 3 \mathrm{~km} \mathrm{~s}^{-1}\right)$, and HD $109995\left(v \sin i=27 \pm 3 \mathrm{~km} \mathrm{~s}^{-1}\right)$. Peterson (1983) used the cross-correlation technique to find these values, after a calibration with an artificially broadened spectrum of a non rotating HB field star, which was also chosen as the template (see Peterson 1983, Sect. IVe). The resolution of her observations was $8.5 \pm 0.5 \mathrm{~km} \mathrm{~s}^{-1}$.
The spectra were extracted manually, trying to achieve the highest possible signal-to-noise. Standard IRAF procedures were used. For the wavelength calibration, ThAr spectra were obtained at the beginning and the end of each night. Thanks to its technical design, UVES is an instrument with a high stability and repeatability of calibrations both over short and extended periods of time. In addition, the spectrograph is placed at the Nasmyth platform minimizing flexure problems. Changes within a night of the atmospheric pressure and the temperature inside the enclosure were always smaller than $1 \mathrm{hPa}$ and $1.5 \mathrm{degC}$ respectively, so the corresponding induced errors are $<50 \mathrm{~m} \mathrm{~s}^{-1}$ and $<250 \mathrm{~m} \mathrm{~s}^{-1}$ (see UVES user manual). This is empirically confirmed by the ThAr spectra taken at the beginning and the end of each night. The seeing disk values oscillated in the range $\sim 0.5 \operatorname{arcsec}$ to $\sim 1.4$ arcsec. As a consequence, the possibility that apparent stellar radial velocity could depend a little on the star's position within the slit has to be taken into account, when the seeing profile was narrower than the slit. On the other hand, the wind speed was always $\leq 10 \mathrm{~m} \mathrm{~s}^{-1}$ so possible errors derived from guiding drift are always very small (a few hundreds of meters per second, see VLT documentation). Moreover, empirical tests on radial velocity performed on our data (cf. Sect. 3) confirm that there are not large $v_{\text {rad }}$ errors due to guiding drift.

We fitted 4th order polynomials to the dispersion relations of the ThAr calibration spectra, which resulted in residuals $\leq 3 \times 10^{-4} \AA$. A mean of 4 to 5 lines per order were used for the polynomial wavelength solution, covering properly all the 31 orders. Each spectrum was divided into sections of about $40 \AA$, which, avoiding hydrogen lines, in general coincided with the echelle UVES orders. We also avoided the broad CaII K line at $3933 \AA$. Finally, each spectrum was normalized using a manual polynomial fitting.

\section{Cross-correlation analysis}

We determined the radial velocity $\left(v_{\mathrm{rad}}\right)$ and the projected rotational velocity $(v \sin i)$ for each of our program stars using the cross-correlation technique described by Tonry \& Davis (1979, TD79). This method is well suited to measure $v_{\text {rad }}$ and rotational broadening in low signal-to-noise spectra (Dubath et al. 1990). The analysis procedure computes (in the Fourier domain) the cross-correlation function (CCF) of the object spectrum versus that of a template, fits a Gaussian to the highest peak, and finds the radial velocity and the line broadening from the peak's central position $(\delta)$ and width $(\sigma)$. If both spectra are very narrow-lined, there is little tolerance for radial velocity displacements, and the peak falls off rapidly; if one or the other is somewhat broad-lined, the falloff is gradual, and the correlation peak is broader. The cross-correlation procedure was performed using the task fxcor within IRAF. For the Gaussian fit of the CCF peak the parameters used were the center position, amplitude and width. Separate CCFs were computed for each spectral order. The heliocentric correction for each spectrum was also computed on the basis of its observational coordinates and time. We used the slow rotating $\left(v \sin i<6 \mathrm{~km} \mathrm{~s}^{-1}\right)$ blue HB field stars HD $74721\left(v_{\text {rad }}=30.7 \mathrm{~km} \mathrm{~s}^{-1} \pm 0.6 \mathrm{~km} \mathrm{~s}^{-1}\right)$ and HD $130095\left(v_{\mathrm{rad}}=66.0 \mathrm{~km} \mathrm{~s}^{-1} \pm 0.7 \mathrm{~km} \mathrm{~s}^{-1}\right)$ as templates for 
the cross-correlation. Those CCFs for which the peak's central position did not agree with the correct stellar radial velocity, relative to the template, were rejected. In general, we considered only those CCFs giving an object $v_{\text {rad }}$ in the range $v_{\mathrm{rad}_{\mathrm{GC}}} \pm 50 \mathrm{~km} \mathrm{~s}^{-1}$, where $v_{\mathrm{rad}_{\mathrm{GC}}}$ is the radial velocity of the corresponding globular cluster, as taken from Harris (1996) compilation. In this way, we avoided the errors due to line mismatch or to the lack of spectral features, especially for the hottest stars and the reddest sections of the spectra. A mean of 15 different CCFs could be used for each program star, each of them providing a value of $\delta$ and $\sigma$. In addition, using $\delta$ as the center position, the antisymmetric part of the correlation function is calculated, and its rms $\left(\sigma_{a}\right)$ is derived. This allows the calculation of the velocity error $(\epsilon)$ via the " $r$ " parameter, as described by TD79:

$$
\begin{aligned}
& r=h /\left(\sqrt{2} \sigma_{a}\right) \\
& \epsilon=N /(8 B(1+r))
\end{aligned}
$$

where $h$ is the height of the cross-correlation peak, $N$ is the number of bins in which the spectrum is mapped (2048 in our case) and $B$ is the highest wave number where the Fourier transform of the CCF has appreciable amplitude (e.g. the halfmaximum point).

To calculate the radial velocity of each program star, we computed the mean $\left(\delta_{\text {mean }}\right)$ of the $\delta$ values of all the good CCFs involving that star, weighted to the inverse of their errors $(1 / \epsilon)$. The final $v_{\text {rad }_{o}}$ of the object star is:

$v_{\text {rad }_{\mathrm{o}}}=v_{\mathrm{rad}_{\mathrm{t}}}+\delta_{\text {mean }}$

where $v_{\mathrm{rad}_{\mathrm{t}}}$ is the most recent radial velocity of the template taken from the literature (Kinman et al. 2000). Hence, the error:

$$
\epsilon\left(v_{\mathrm{rad}_{\mathrm{o}}}\right)=\sqrt{\epsilon^{2}\left(v_{\mathrm{rad}_{\mathrm{t}}}\right)+\epsilon^{2}\left(\delta_{\text {mean }}\right)}
$$

where $\epsilon\left(v_{\mathrm{rad}_{\mathrm{t}}}\right)$ is taken from Kinman et al. (2000) and $\epsilon\left(\delta_{\text {mean }}\right)$ is the rms scatter of the individual $\delta$ values. In order to take into account possible systematic errors on the $v_{\text {rad }}$ measurements induced by the adopted values of $v_{\mathrm{rad}_{\mathrm{t}}}$, we have tested the internal consistency among the field stars used. Cross-correlations among all the spectra of the stars HD 74721 and HD 130095 observed (seven spectra for each star) confirm an agreement with Kinman et al. (2000) inside the errors and a consistency of $<0.6 \mathrm{~km} \mathrm{~s}^{-1}$ among the standards.

Similarly, after the appropriate calibration, we obtained the $v \sin i$ for each target from the value of $\sigma$, as explained in the following section. Figure 2 shows an example of the CCFs obtained and fitted. The target star is n2808-2333, and the template is HD 74721. The corresponding normalized spectra sections are presented in Fig. 3. The background level is always fixed at the zero value, on the complete range of the CCF to avoid the sensitivity of the results to details of the shape of the correlation function.

\section{Projected rotational velocity measurements}

The width $(\sigma)$ of the CCF of a star results from several broadening mechanisms which depend on, for instance, gravity, turbulence, magnetic fields, effective temperature, metallicity, rotation. In addition, the instrumental profile also contributes to the broadening of the spectrum and therefore to the CCF. Thus, in order to correctly measure the rotational contribution to the width of the $\mathrm{CCF}$, we must model the contributions of the broadening mechanisms other than rotation, that is, calibrate the $\sigma-v \sin i$ relation for the instrument and the stars we are working with. In the case of a Gaussian fit of the CCF peak, the rotational broadening will correspond to a quadratic broadening of the CCF:

$\sigma^{2}=\sigma_{\text {rot }}^{2}+\sigma_{\mathrm{o}}^{2} \Rightarrow \sigma_{\text {rot }}^{2}=\sigma^{2}-\sigma_{\mathrm{o}}^{2}$

where $\sigma$ is the width of the CCF, $\sigma_{\text {rot }}$ is the rotational broadening, and $\sigma_{\mathrm{o}}$ gives the non rotational contributions to the width of the CCF from both the object star and from the template, which we suppose to be equal (same spectral type and same instrumental broadening). Therefore, the projected rotational velocity $v \sin i$ is given by (Benz \& Mayor 1984):

$v \sin i=A \cdot \sqrt{\sigma^{2}-\sigma_{\mathrm{o}}^{2}}=A \cdot \sigma_{\mathrm{rot}}$

where $A$ is a constant coupling the differential broadening of the CCF to the difference in $v \sin i$ between the template and the object. The value of $A$ in the previous equation was found by fitting a straight line to the relation, $v \sin i$ versus $\sigma_{\text {rot }}$, for all the field standards of rotation, assuming $3 \mathrm{~km} \mathrm{~s}^{-1}$ for the rotation of the templates. The slope gives $A$. The best fit of the points, $\langle A\rangle=1.8 \pm 0.3$, was adopted (see Fig. 4 ). The previous relation was observed to be linear in our range of interest, and even for faster rotation values, by Lucatello \& Gratton (2003, see their Fig. 2). This is also confirmed by the very low residuals $(<0.1)$ that we find by fitting the cross-correlation peaks with a Gaussian profile.

As explained by Melo et al. (2001), there is also a smooth dependence on $\sigma_{\mathrm{o}}$ on the stellar colour and luminosity class. However, the variation of $\sigma_{\mathrm{o}}$ within the range of our program and template stars is expected to be quite small compared to the accuracy of our $\sigma$ measurements $\left(\sim 3-6 \mathrm{~km} \mathrm{~s}^{-1}\right)$, due to the relatively small number of lines and the low $S / N$ for our spectra. Moreover, we have an error of $3 \mathrm{~km} \mathrm{~s}^{-1}$ in the $v \sin i$ values of the templates. For this reason, we decided to estimate the value of $\sigma_{\mathrm{o}}$ by correlating one template with the other, for each one of the orders used to measure the $v \sin i$ of the target stars. In other words, $\sigma_{\mathrm{o}}^{2}$ is equal to $\tau_{1}^{2}+\tau_{2}^{2}$, where $\tau_{1}$ and $\tau_{2}$ are the line widths of the templates, in this case HD 74721 and HD 130095. The value of $\sigma_{\mathrm{o}}$ changes very little with the echelle order used or the night of observation, and it has a mean value of $13.3 \pm 0.4 \mathrm{~km} \mathrm{~s}^{-1}$. In order to test the consistency of the previous approximations, we have estimated the values of $\tau_{1}$ and $\tau_{2}$ from the autocorrelations of HD 74721 and HD 130095. As in the case of $\sigma_{\mathrm{o}}, \tau_{1}$ and $\tau_{2}$ are quite constant with the order and the night of observation and the mean values determined are $\tau_{1}=12.8 \pm 0.3 \mathrm{~km} \mathrm{~s}^{-1}$ and $\tau_{2}=13.3 \pm 0.6 \mathrm{~km} \mathrm{~s}^{-1}$. To avoid an underestimation on the error, we have cross-correlated different spectra of the same template, instead of autocorrelating an spectrum with itself. These results suggest that the $v \sin i$ value is quite the same for both templates, specially considering their similar atmospheric parameters (Kinman et al. 2000). Nevertheless, in the case of the hotter targets in our sample, we could be slightly overestimating their rotational rates if their $\sigma_{\mathrm{o}}$ 

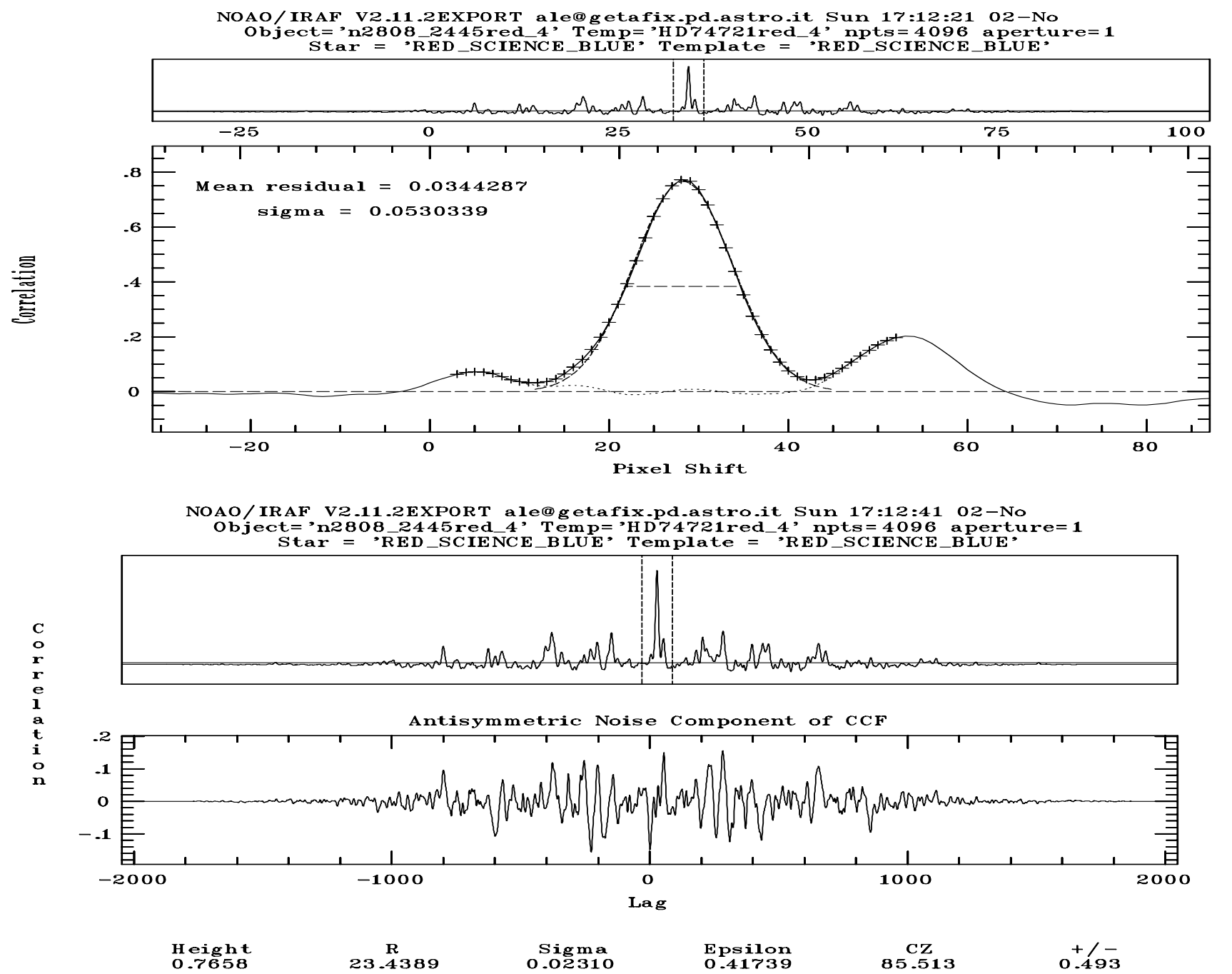

Fig. 2. Example of a cross-correlation function (top panel), a zoom of the peak (central panel) and the corresponding antisimmetric part (bottom panel). The target star is $\mathrm{n} 2808-2333$ and the template is HD 74721. The values of the $r, \epsilon, \sigma_{a}$ parameters are also provided.

were somewhat larger than the one of our templates. However, for stars with temperatures $>11500 \mathrm{~K}$, the absence of microturbulence and surface convection could, at least partially, compensate the previous effect. In any case, the overall effect is expected to be rather small compare to the accuracy of our measurements.

In order to derive the final $v \sin i$ for each program star, we calculated the mean of the $v \sin i$ values given by the individual $\sigma$ determinations for all the good CCFs (15 on average) involving that star. We used as weight the height of the crosscorrelation peak.

The error of each $v \sin i$ measurement can be obtained by differentiating Eq. (1):

$|\Delta(v \sin i)|_{\mathrm{mes}}=A^{2} \sqrt{(\epsilon \sigma)^{2}+\left(\epsilon_{\mathrm{o}} \sigma_{\mathrm{o}}\right)^{2}} / v \sin i$

where $\epsilon$ and $\epsilon_{\mathrm{o}}$ are the velocity errors for the CCF and the template to template CCF respectively. To this we have to add the systematic errors due to the uncertainty in the templates and the standards, which also affect the calibration curve. A first systematic error, $|\Delta(v \sin i)|_{t}$, has been evaluated by re-calculating the $v \sin i$ of each object star assuming a $v \sin i$ for the templates of $0 \mathrm{~km} \mathrm{~s}^{-1}$ and $6 \mathrm{~km} \mathrm{~s}^{-1}$. A second systematic, $|\Delta(v \sin i)|_{A}$, error was derived by re-calculating the $v \sin i$ of the targets with A set to its $+\sigma$ and $-\sigma$ values. Thus, the total error on $v \sin i$ is:

$|\Delta(v \sin i)|_{\mathrm{tot}}=\sqrt{|\Delta(v \sin i)|_{\mathrm{mes}}^{2}+|\Delta(v \sin i)|_{t}^{2}+|\Delta(v \sin i)|_{A}^{2}}$.

\section{Results}

The final results for a dataset of 61 stars in the clusters NGC 1904 (M 79), NGC 2808, NGC 6093 (M 80) and NGC 7078 (M 15) are presented in Tables 4 and 5. For each target star (Col. 1), the radial velocity (Col. 2), and the projected rotational velocity (Col. 3) are given. The three sources of error (random error, error due to the templates uncertainty, and error due to the calibration curve) are presented in Cols. 4, 5 and 6. The total error, resulting from the quadratic addition of random and systematic errors is given in Col. 7. The M 79 Strömgren photometric data from G99 have been kindly provided by Grundahl. The NGC $2808 U, U-B$ photometry is from Bedin et al. (2000). The M 80 and M 15 photometry comes from Piotto et al. (2002), except for 5 stars in M 80 and 3 stars 


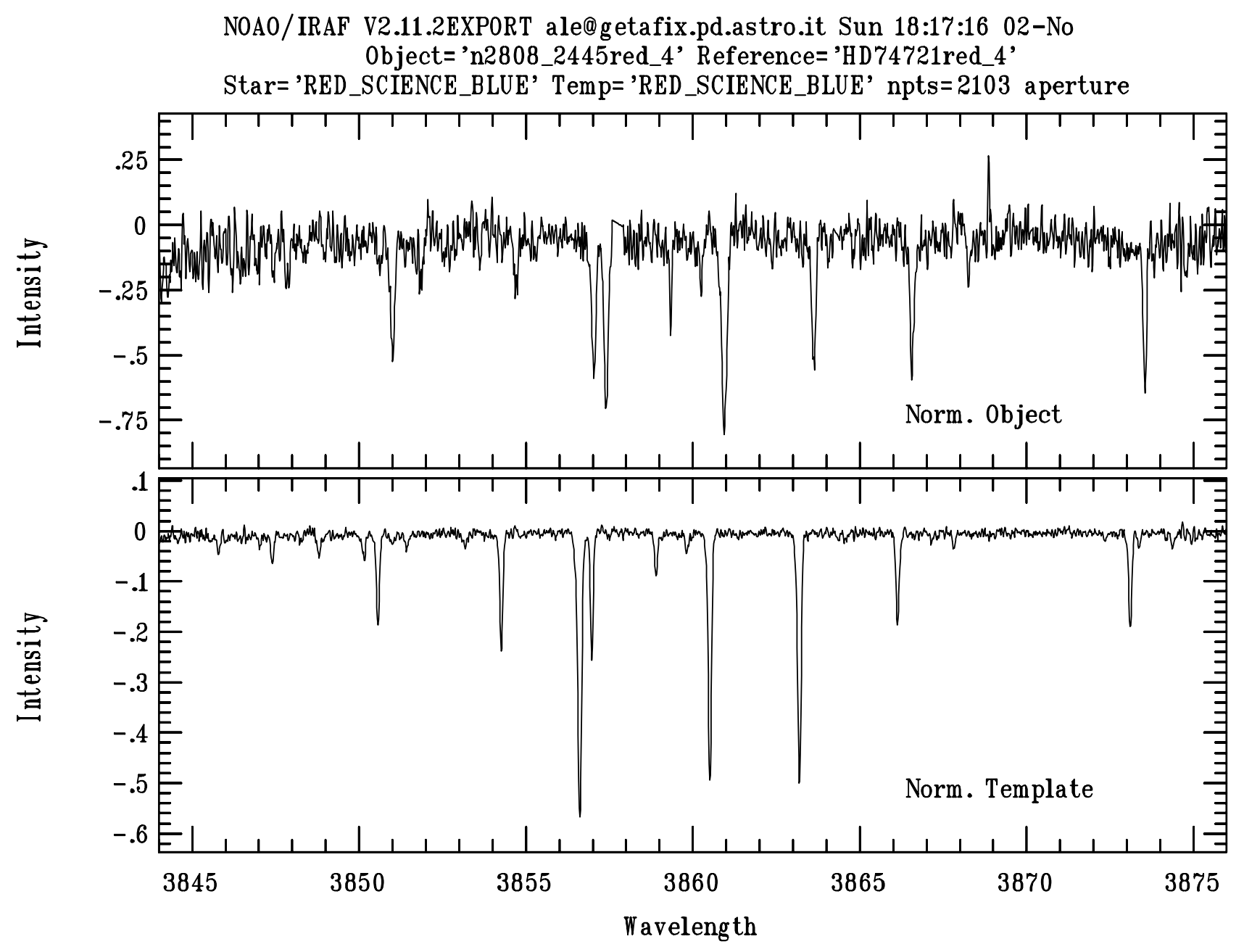

Fig. 3. Normalized section of the spectrum of the target star n2808-2333 (top panel) and the template HD 74721 (bottom panel).

in M 15, taken from Rosenberg et al. (2000). In addition, the effective temperature has been derived by comparing the Cassisi et al. (1999) models with the corresponding colour-magnitude diagrams. We tested the consistency of the derived $T_{\text {eff }}$ values from different photometric colours comparing the Strömgren photometry of cluster M 79 with a Johnson photometry by Momany et al. (2003, private communication). The temperature differences were always $\leq 1000 \mathrm{~K}$.

The stars $\mathrm{m} 15-\mathrm{b} 130$ and $\mathrm{m} 15-\mathrm{b} 218$ are in common with B00b. Our $v \sin i$ measurements and B00b values for this two stars are in agreement within the errors $(5 \pm 3$ vs. $5.07 \pm 0.24$ and $13 \pm 3$ vs. $14.88 \pm 0.69$ ), which reveals the consistency between both observations and the two independent methods applied for the rotational velocity measurement (B00 used a profile fitting method).

Figure 4 shows the complete set of projected rotational velocity data from 5 BT Galactic GCs: M 79, NGC 1904, M 80, M 15 and M 13. Besides the data from this paper (circles), Fig. 4 includes the B00b data for M 15 (open triangles), plus the B00a (full triangles) and Peterson et al. (1995, full squares) measurements for M 13.

As already pointed out in R02, all the stars hotter than $T_{\text {eff }} \sim 11500 \mathrm{~K}$ have $v \sin i \leq 12 \mathrm{~km} \mathrm{~s}^{-} 1$, indicating that the bulk of these stars must be intrinsically slow rotators. At
$T_{\text {eff }} \sim 11500 \mathrm{~K}$ (which is the temperature of the $u$-jump of G99) there is an abrupt change in the rotational velocity distribution. Among the cooler stars $\left(T_{\text {eff }}<11500 \mathrm{~K}\right)$, there is a range of rotation rates, with a group of stars rotating at $\sim 15 \mathrm{~km} \mathrm{~s}^{-1}$ or less, and a fast rotating group at $\sim 30 \mathrm{~km} \mathrm{~s}^{-1}$. A few stars reach a projected rotation velocity of about $40 \mathrm{~km} \mathrm{~s}^{-1}$. As noticed by $\mathrm{R} 02$, the fraction of fast rotators is different in different clusters (cf. Figs. 1 and 2 in their paper), with fast rotators relatively more abundant in M 13 and M 79 than in the other clusters of the present sample. In M 13 and M 79, at least half of the stars cooler than $T_{\text {eff }}=11500 \mathrm{~K}$ are fast rotators, while only 3 out of 22 stars rotate faster than $15 \mathrm{~km} \mathrm{~s}^{-} 1$ in M 15. At the present time, no fast rotators have been identified in NGC 2808 and M 80. Figure 5 shows the projected rotational velocities for the 16 target stars in NGC 2808, with the new stars presented in this paper added. All the newly observed stars are cooler than $T_{\text {eff }}=11500 \mathrm{~K}$. Again, none of them has a projected rotation velocity higher than $13 \mathrm{~km} \mathrm{~s}^{-1}$. However, on the cool side of the gap, we have rotation velocities for only 9 stars in NGC 2808 and 7 stars in M 80. If the fraction of fast rotators in these two clusters is as low as in M 15 (or lower), we might have missed them because of the small number of observed targets. The rotation rate distribution, and its variation from cluster to cluster must be studied. The advent of the 


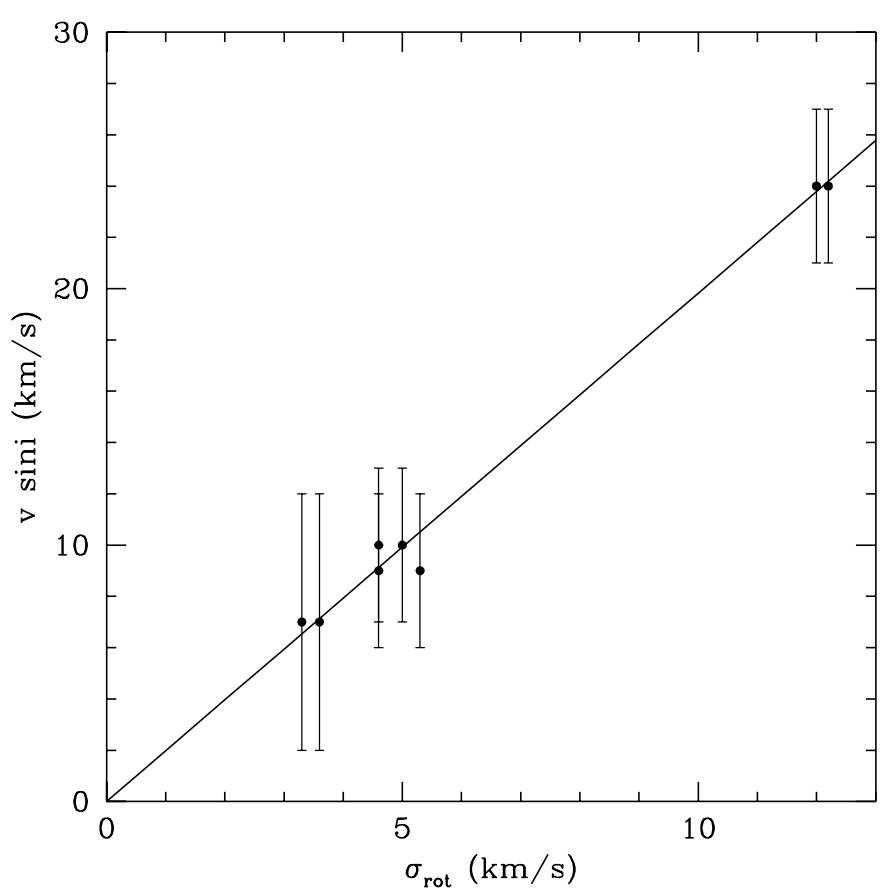

Fig. 4. Calibration of the differential broadening of the CCF to the projected rotational velocity $v \sin i$ for the standard stars taken from Peterson et al. (1983).

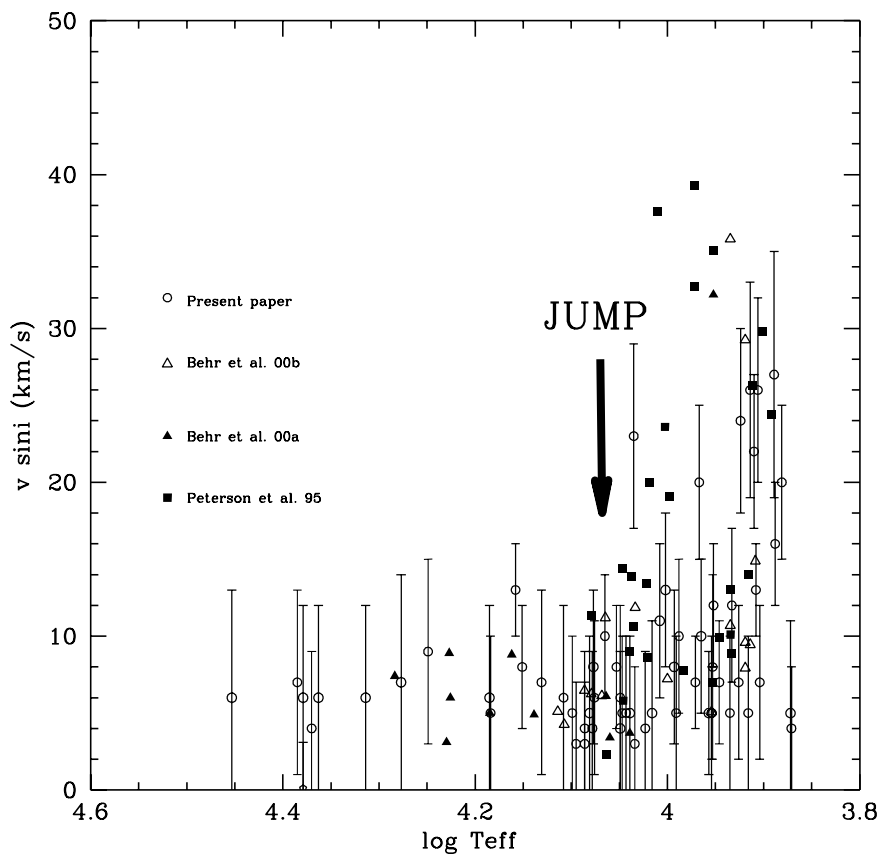

Fig. 5. Projected rotational velocities as a function of the temperature for all our 61 targets (open circles), plus the B00b (open triangles), B00a (full triangles) and Peterson et al. (1995, full squares) meassurements in M 15 and M 13. The vertical arrow indicates the position of the "jump" (from G99) in correspondence of $T_{\text {eff }}=11500 \mathrm{~K}$ where all the HBs of G99 GCs show this feature.

multifiber spectrograph FLAMES at VLT will provide a unique opportunity to carry out this project. In particular, we plan to continue our study with new observations to increase statistics of rotational velocity measurements.

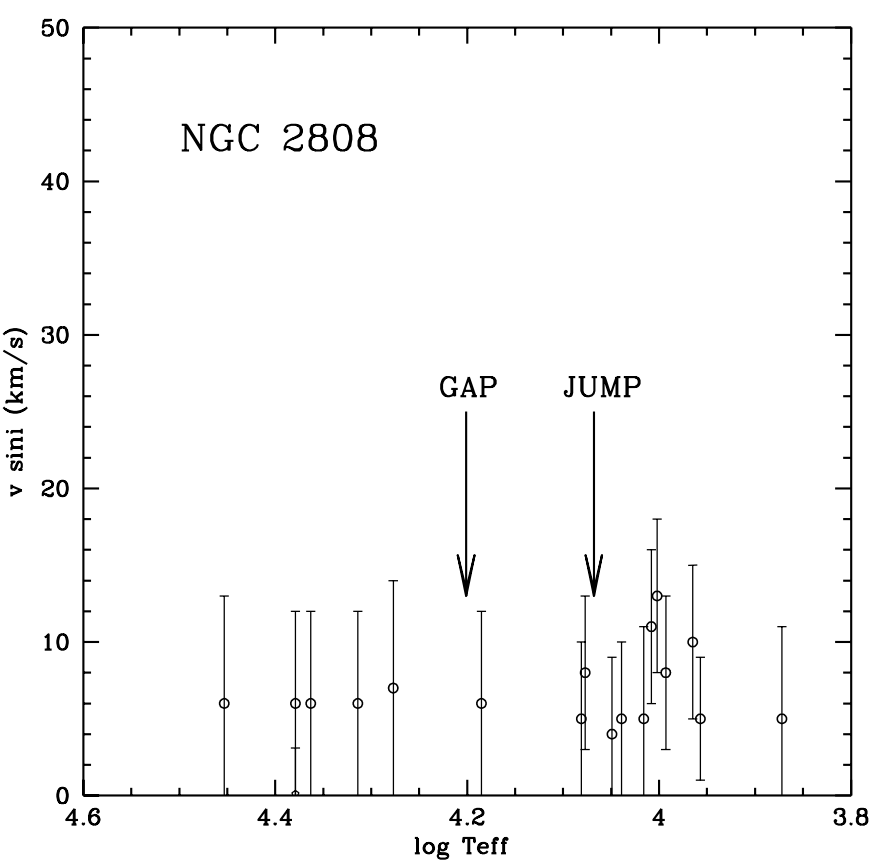

Fig. 6. Projected rotational velocities as a function of the temperature for NGC 2808.

Figure 4 clearly suggests a link between the photometric G99 jump and the discontinuity in the distribution of the HB stellar rotation rates. Radiative levitation of metals has been invoked by G99 to explain the jump. Besides, the metal abundance anomalies (enhancements of metals, underabundance of $\mathrm{He}$ ) found by Behr et al. (1999) and B00b constitute an empirical evidence that radiative levitation and diffusion are effectively at work in the envelope of HB stars with $T_{\text {eff }} \geq 11500 \mathrm{~K}$. As a consequence, R02 argue that the absence of fast rotators at $T_{\text {eff }} \geq 11500 \mathrm{~K}$ might be due to an increase of angular momentum removal caused by enhanced mass loss in the more metallic atmospheres of the stars hotter than the jump. An enhanced mass loss in higher metallicity atmospheres has been very recently calculated by Vink \& Cassisi (2002), confirming this scenario. Radiative levitation and the "wind emission" effect is also the most likely explanation for the observed jump in the stellar gravities (Moehler et al. 2000; Vink \& Cassisi 2002).

However, the real open problem is that we still lack of any plausible explanation for the presence of fast rotators (see discussion in R02).

Acknowledgements. We thank L. R. Bedin, F. Grundahl and A. Rosenberg for providing their photometries. We are also grateful to S. Lucatello for useful discussions. We sincerelly thank the anonymous referee for the care and the interest dedicated to this work and the important contribution to the improvement of this paper. A.R.B. recognizes the support of the Istituto Nazionale di Astrofisica (INAF). G.P. recognizes partial support from the Ministero dell'Istruzione, Università e Ricerca $(M I U R)$, and from the Agenzia Spaziale Italiana $(A S I)$. 


\section{References}

Bedin, L. R., Piotto, G., Zoccali, M., et al. 2000, A\&A, 363, 159 Behr, B. B., Cohen, J. G., McCarthy, J. K., \& Djorgovski, S. G. 1999, ApJ, 517, L31

Behr, B. B., Djorgovski, S. G., Cohen, J. G., et al. 2000a, ApJ, 528, 849 [B00a]

Behr, B. B., Cohen, J. G., \& McCarthy, J. K. 2000b, ApJ, 531, L37 [B00b]

Benz, W., \& Mayor, M. 1984, A\&A, 138, 183

Cassisi, S., Castellani, V., degl'Innocenti, S., Salaris, M., \& Weiss, A. 1999, A\&AS, 134, 103

Catelan, M., Borissova, J., Sweigart, A. V., \& Spassova, N. 1998, ApJ, 494, 265

Dubath, P., Meylan, G., Mayor, M., et al. 1990, A\&A, 239, 142

Grundahl, F., Catelan, M., Landsman, W. B., Stetson, P. B., \& Andersen, M. I. 1999, ApJ, 524, 242 [G99]

Harris, W. E. 1996, AJ, 112, 1487

Lucatello, S., \& Gratton, R. G. 2003, A\&A, 406, 691
Melo, C. H. F., Pasquini, L., \& De Medeiros, J. R. 2001, A\&A, 375, 851

Moehler, S., Sweigart, A. V., Landsman, W. B., \& Heber, U. 2000, A\&A, 360, 120

Momany, Y. 2003, private communication

Peterson, R. C. 1983, ApJ, 275, 737

Peterson, R. C. 1985a, ApJ, 289, 320

Peterson, R. C. 1985b, ApJ, 294, 35

Peterson, R. C., Rood, R. T., \& Crocker, D. A. 1995, ApJ, 453, 214

Piotto, G., Zoccali, M., King, I. R., et al. 1999, AJ, 118, 1727 [P99]

Piotto, G., King, I. R., Djorgovski, S. G., et al. 2002, A\&A, 391, 945

Recio-Blanco, A., Piotto, G., Aparicio, A., \& Renzini, A. 2002, ApJ, 572,71 [R02]

Renzini, A. 1977, in Advanced Stages in Stellar Evolution (Geneva Observatory), 149

Rosenberg, A., Piotto, G., Saviane, I., \& Aparicio, A. 2000, A\&AS, 144,5

Tonry, J., \& Davis, M. 1979, AJ, 84, 1511

Vink, J.-S., \& Cassisi, S. 2002, A\&A, 392, 553 